\title{
¿Cómo se construye la convivencia escolar en la educación Montessori? Estudio de caso de una escuela Montessori de Valparaíso, Chile
}

\author{
How is the school coexistence constructed in the Montessori education? \\ A case study from a Montessori school in Valparaíso, Chile
Karen Cuevas Solís ${ }^{a}$, Betzabé Álvarez Palta ${ }^{a}$,Ewerton Cortés Bernal, Franco Passi Rojas ${ }^{a}$, Felipe Apablaza Campos ${ }^{a}$ \\ ${ }^{a}$ Universidad de Playa Ancha, Valparaíso, Chile. \\ karen.cuevas@upla.cl
}

\begin{abstract}
RESUMEN
La educación Montessori es un enfoque pedagógico que se centra en el desarrollo integral del niño ${ }^{1}$, quien debe construir su propio aprendizaje. Dentro de este enfoque, el compromiso del individuo hacia el grupo resulta fundamental, por lo que es crucial una apropiada convivencia escolar. Investigaciones internacionales sugieren que el paradigma de la Justicia Restaurativa es exitoso para mejorar la convivencia escolar. Esta investigación cualitativa busca indagar y recoger elementos que permitan establecer cómo se construye la convivencia escolar en una escuela Montessori de Valparaíso, Chile, a través de percepciones de los niños y maestros. Los hallazgos señalan que los aspectos más importantes son: el desarrollo de la autonomía, adaptabilidad y autoestima por parte de los niños; el rol pasivo del maestro Montessori; y la resolución de conflictos, mediante una estrategia restaurativa denominada "Círculo de Emergencia".
\end{abstract}

Palabras claves: Educación Alternativa, Resolución de Conflicto, Justicia Restaurativa, Enfoque Montessori.

\begin{abstract}
Montessori education is a pedagogical perspective centered in the integral development of children, who must construct their own learning. In this perspective, the engagement of the individual to the group is fundamental. For that reason, an appropriated school coexistence is crucial. International research suggests that Restorative Justice paradigm is a successful way to improve the school coexistence. This qualitative research seeks to investigate and collect elements that allow establishing how it is built the school coexistence in a Montessori school from Valparaíso, Chile, through perceptions from children and teachers. The findings emphasize the main aspects which are the development of autonomy, adaptability and self-esteem by children; the passive role of Montessori teacher; and the conflict resolution by a restorative strategy named "Círculo de Emergencia".
\end{abstract}

Key words: Alternative Education, Conflict Resolution, Restorative Justice, Montessori Perspective.

\footnotetext{
En este artículo se utilizan de manera inclusiva términos como "maestra", "niño", y sus respectivos plurales (así como otras palabras equivalentes en el contexto educativo) para referirse a hombres y mujeres. Esta opción obedece a que no existe acuerdo universal respecto de cómo aludir conjuntamente a ambos sexos en el idioma español, salvo usando "o/a", "los/las" y otras similares, y ese tipo de fórmulas supone una saturación gráfica que puede dificultar la compresión de la lectura.
} 
Estudios Pedagógicos XLVII N²: 299-317, 2021

¿CÓMO SE CONSTRUYE LA CONVIVENCIA ESCOLAR EN LA EDUCACIÓN MONTESSORI? ESTUDIO DE CASO DE

UNA ESCUELA MONTESSORI DE VALPARAÍSO, CHILE

\section{INTRODUCCIÓN}

La convivencia escolar ha sido un asunto a tratar a nivel internacional, tanto a través de políticas públicas como por iniciativas de instituciones particulares. La convivencia se fundamenta en diversos elementos, como los valores de pluralidad, participación democrática, inclusión social y respeto a la diferencia, igualdad de oportunidades, manejo positivo de los conflictos y desarrollo de una cultura para la paz. Para ello, es necesario la conciencia de la propia identidad, la aceptación del otro y un sentido de pertenencia y contribución (Generalitat de Catalunya, 2017). Estos mismos elementos se extrapolan al contexto educativo y deben ser incorporados al proyecto de toda escuela (Muñoz et al., 2014).

El conflicto se entiende como una circunstancia donde las personas contienden con otras por la consecución de uno o varios objetivos. Esto puede implicar desarmonía, incompatibilidad y pugna entre dos o más partes independientes (Funes, 2000). Muñoz (2004) sugiere que el conflicto es necesario desde un punto de vista social, dado que ha permitido interpretar a las personas y los grupos sociales. También indica que, de no ser por los conflictos, la sociedad no hubiese alcanzado el desarrollo intelectual para las habilidades sociales, regulación de la conducta, creatividad, etc.

El conflicto puede abordarse desde dos enfoques: (1) formativo, donde se invita a la reflexión y a la resolución pacífica de la pugna; y (2) punitivo, donde se busca la sanción y contiene una mirada castigadora de los problemas de convivencia (Carrasco et al., 2018). Las medidas punitivas han demostrado ser ineficientes, además de provocar un impacto negativo en la comunidad escolar. A pesar de ello, muchas escuelas las utilizan como un fin para el control social (Morrison y Vaandering, 2012). En contraposición, un enfoque formativo tiene un efecto positivo dentro de las comunidades educativas. El conflicto es valorado como una oportunidad para el aprendizaje de habilidades sociales (Gellin, 2018). La aplicación del paradigma de la Justicia Restaurativa en escuelas es utilizada como una medida formativa de la resolución de conflictos. Este paradigma toma como elemento clave el compromiso social, que permite enriquecer el ambiente escolar y los lazos sociales subyacentes. Se busca reparar el daño y crear oportunidades. Dentro de los beneficios que ofrece este enfoque, está fortalecer el derecho de los niños a ser escuchados y participar, incrementar la democracia en los espacios educativos, promover las habilidades sociales de los estudiantes, incrementar su capacidad de tomar responsabilidades, crear un ambiente de paz, prevenir futuros problemas de conducta y disminuir la exclusión social (Gellin, 2018; Morrison \& Vaandering, 2012).

\subsection{ESTRATEGIAS RESTAURATIVAS DE RESOLUCIÓN DE CONFLICTO}

Es frecuente encontrar establecimientos que focalizan sus prácticas en casos puntuales de problemas de comportamiento (por lo general de los estudiantes), pero que no presentan una visión más integral sobre cómo mejorar la convivencia, en la cual no solo se incluya a los estudiantes, sino que al resto de la comunidad educativa (Carrasco et al., 2018). Se requiere del desarrollo e implementación de un reglamento interno participativo que permita afrontar diferentes situaciones donde se trasgredan las normas de convivencia, permitiendo no solo enfrentarlas de forma propositiva y constructiva, sino que también prevenirlas (Muñoz et al., 2014). 
Desde el paradigma de la Justicia Restaurativa se ofrecen diversas estrategias que pueden ser utilizadas en la resolución de conflictos, dentro de las cuales se encuentran el arbitraje, la mediación, el diálogo y los círculos de paz (Acosta, 2004; Morrison \& Vaandering, 2012). El arbitraje es un proceso donde dos o más partes se encuentran frente a una disputa e invitan a un tercero a resolver la situación, ya que no lo pueden hacer por sí mismos. Este árbitro debe mantenerse neutral para tomar una decisión imparcial, la cual debe ser obedecida (Acosta, 2004; Badiei, 2010). Por otro lado, la mediación es una negociación donde las partes en conflicto invitan a un mediador, quien cumple un rol de intermediario neutral. Este debe permitir que exista una comunicación efectiva entre las partes, fomentar los lazos y realizar sugerencias para que ellos mismos sean quienes establezcan qué acciones y compromisos seguirán para solucionar el problema (Acosta, 2004; Morrison \& Vaandering, 2012). El diálogo es una estrategia que involucra a las partes implicadas, así como también a profesionales y personas de apoyo (Morrison \& Vaandering, 2012). Se busca llegar a un acuerdo común, explicando y defendiendo sus posiciones y sentimientos, con el fin de encontrar una solución útil y que ayude a que la relación perdure, además de cultivar la autosuficiencia para solucionar problemas. Para ello, es importante que las partes estén dispuestas a escuchar a los otros y no sólo seguir intereses propios (Acosta, 2004). Por último, los círculos de paz son un proceso proactivo de resolución, donde cada uno de los participantes de la comunidad puede hablar del problema desde su punto de vista y las soluciones que se proponen para, en grupo, llegar a un consenso. De esta forma, se generan enriquecimiento social y emocional dentro de la comunidad, donde se destaquen valores tales como la honestidad, la inclusión, la empatía, el perdón, etc. (Morrison \& Vaandering, 2012).

\subsection{EXPERIENCIAS DE CONVIVENCIA ESCOLAR}

A continuación, presentamos algunos ejemplos de convivencia escolar en el mundo. En Finlandia se realiza el programa VERSO desde el año 2000, actualmente bajo la dirección del Finnish Forum for Mediation y el Ministry of Social Affairs and Health. Este programa se fundamenta bajo el paradigma de la Justicia Restaurativa, utilizando la mediación como método de la resolución de conflicto en las escuelas. Según Gellin (2018), el objetivo del programa es incrementar la democracia y la participación de los alumnos, fortalecer las relaciones y mejorar el aprendizaje de habilidades sociales para la resolución de conflictos, no solo para el ámbito escolar, sino que para la vida en comunidad. Los resultados de la evaluación y análisis del programa realizado el año 2016 indican que el $60 \%$ de los participantes considera muy beneficioso el conocimiento y aplicación de la mediación y el $71 \%$ considera que ha mejorado la convivencia escolar disminuyendo las situaciones disruptivas, puesto que, del total de casos, el $82 \%$ han sido resueltas a través de esta forma (Gellin, 2018).

En Estados Unidos también han existido iniciativas de diversas instituciones educativas donde se ha aplicado el paradigma de la Justicia Restaurativa para la resolución de los conflictos (Fronius et al., 2016; Gellin, 2018). En la década de 1980, las políticas de Tolerancia Cero comenzaron a ser populares frente a las conductas disruptivas, provocando un impacto negativo en los estudiantes (Losen, 2014, citado en Fronius et al., 2016). Estas se han incrementado por actos terroristas, como tiroteos en escuelas y los eventos del 9/11 (Morrison \& Vaandering, 2012), incluso siendo aplicadas de forma discriminatoria frente a 
Estudios Pedagógicos XLVII N² 2: 299-317, 2021

¿CÓMO SE CONSTRUYE LA CONVIVENCIA ESCOLAR EN LA EDUCACIÓN MONTESSORI? ESTUDIO DE CASO DE UNA ESCUELA MONTESSORI DE VALPARAÍSO, CHILE

estudiantes de minorías étnicas o raciales (Fabelo et al., 2011). Tras esto, diversas escuelas cambiaron sus protocolos por una visión restaurativa, obteniendo los resultados que se resumen en la Tabla 1.

Tabla 1. Implementación de la Justicia Restaurativa en Escuelas Estadounidenses

\begin{tabular}{|c|c|c|}
\hline Ciudad, Estado & Principales Hallazgos & Referencia \\
\hline \multirow{4}{*}{$\begin{array}{l}\text { San Antonio, } \\
\text { Texas }\end{array}$} & $\begin{array}{l}65 \% \text { de los estudiantes disminuyeron las suspensiones } \\
\text { escolares en sexto grado. }\end{array}$ & \multirow{4}{*}{ Armour, 2013} \\
\hline & $\begin{array}{l}72 \% \text { de los estudiantes disminuyeron las suspensiones } \\
\text { escolares en séptimo grado. }\end{array}$ & \\
\hline & $\begin{array}{l}38 \% \text { de los estudiantes disminuyeron la impuntualidad } \\
\text { escolar (primer año de estudio). }\end{array}$ & \\
\hline & $\begin{array}{l}\text { Se favoreció el trato entre el personal de la escuela y los } \\
\text { estudiantes de minorías étnicas y raciales. }\end{array}$ & \\
\hline $\begin{array}{l}\text { Denver, } \\
\text { Colorado }\end{array}$ & $\begin{array}{l}\text { Se redujo el } 50 \% \text { del ausentismo escolar al primer año } \\
\text { de implementación. }\end{array}$ & $\begin{array}{l}\text { Baker, 2009, citado en } \\
\text { Fronius et al., } 2016 .\end{array}$ \\
\hline \multirow[t]{2}{*}{ East Cost } & $\begin{array}{l}\text { Estudiantes consideraron a los profesores más } \\
\text { respetuosos que antes de la aplicación del programa de } \\
\text { Justicia Restaurativa, incluso los estudiantes de } \\
\text { minorías. }\end{array}$ & \multirow[t]{2}{*}{ Gregory et al., 2016.} \\
\hline & $\begin{array}{l}\text { Los profesores percibieron un mayor compromiso por } \\
\text { parte de los estudiantes tras la aplicación del programa } \\
\text { de Justicia Restaurativa. }\end{array}$ & \\
\hline
\end{tabular}

(Fuente: elaboración propia).

En Chile, desde las políticas públicas, todas las escuelas deben implementar Planes de Gestión de la Convivencia. Estos buscan lograr un clima social nutritivo, instalando la problematización de la convivencia y, de esta forma, avanzar hacia la implementación de procesos efectivos de gestión (Aron et al., 2017). Estos autores señalan que el clima de convivencia escolar es un tema internalizado por los establecimientos educacionales chilenos y que estos se caracterizan por contar con instrumentos de gestión del clima escolar, como protocolos y manuales. Un estudio realizado por la Agencia de la Calidad de la Educación (2013) recolectó las percepciones de los estudiantes de segundo medio sobre conductas de agresión en sus escuelas, a partir del Cuestionario Estudiante SIMCE Segundo Medio 2012 e información sobre los establecimientos a partir de datos administrativos. Los resultados concluyeron que $21,3 \%$ de los establecimientos presentan una alta frecuencia de percepción de conductas agresivas recurrente, 46,2\% presenta una frecuencia media y un $32,5 \%$ presenta una frecuencia baja. Este equipo de investigación plantea que los antecedentes anteriores evidencian que las políticas públicas chilenas son incipientes, cumpliendo solo con protocolos y manuales, de esta forma los esfuerzos se dirigen a la normativa y a su marco regulador, y no a lo esencial de los conflictos de convivencia ¿cómo 
educar en la sana convivencia?, aspecto medular que tienen relación con la formación de un ciudadano íntegro, capaz de resolver conflictos por la vía de la paz.

\subsection{EDUCACIÓN MONTESSORI}

Dentro de los distintos enfoques de enseñanza, la filosofía educativa Montessori presenta elementos de convivencia escolar importantes a destacar, que están en relación a cómo esta concibe la educación. Esta visión se focaliza en el desarrollo del individuo, la cual debe asistir al niño en sus diversos procesos y reforzar las distintas facultades que este posea (Montessori, 2015). Fue fundada por María Montessori, quien, a finales del siglo XIX, desarrolló un método pedagógico para la enseñanza de niños en situación de discapacidad, lo que era abordado de forma médica en la época. Posteriormente, y tras los resultados, decidió aplicar la metodología desarrollada en niños de desarrollo típico, para potenciar sus capacidades. Montessori fue muy crítica en cuanto al mal uso de la ciencia en la educación, proponiendo que el verdadero sujeto de estudio debe ser el niño y su desarrollo, a través de la observación. De esta forma nació lo que ella llamó Método de la Pedagogía Científica, el cual posteriormente fue conocido como Método Montessori (Montessori, 2015; Montessori, 2016b).

Bajo esta mirada, el niño es un sujeto en igualdad de derechos que los adultos y, por tanto, cambia la percepción y el trato que se tiene hacia a ellos (Montessori, 2008; Montessori, 2015). Para el desarrollo del niño, es importante el proceso de normalización, que es entendido como la liberación de las restricciones impuestas por el sistema educativo y es un proceso de preparación previo a la entrega de las herramientas para su desarrollo (Montessori, 2016b). Cuando la normalización se ha logrado, el niño es capaz de desarrollar sus potencialidades, caracterizándose como una persona capaz de concentrarse, ser independiente y libre. La concentración en el trabajo es tomada como un indicador de cambio de personalidad en el niño, dado que comienza a preferir el orden sobre el desorden (Montessori, 2016a). La independencia es concebida como una meta que los niños deben alcanzar tras un proceso de sucesivas conquistas que se llevan a cabo para su desarrollo (Montessori, 2013). Este proceso es ayudado por los adultos, quienes deben guiar al niño en sus conquistas, considerando que cualquier ayuda innecesaria debe ser evitada (Montessori, 2016c). El concepto de la libertad cobra una importancia fundamental, ya que es concebida como la actividad más elevada que el niño desarrolla, la cual es entendida como la capacidad del individuo de identificar qué es lo que necesita y poder realizar las elecciones que le permitan conseguirlo (Montessori, 2015).

\subsubsection{La Escuela Montessori}

La libertad es parte de la estructura de las aulas Montessori, a través de sus puertas abiertas permite la circulación de los estudiantes. Montessori (2016a) considera la circulación como "the utmost importance for the development of a culture" (p. 63). Otra característica importante son las edades de los niños, donde es frecuente encontrar diferentes edades, con un rango de tres años. Esto permite el desarrollo cultural del niño y potenciar la capacidad de aprendizaje y la cooperación para la construcción del mismo, intelectual y socialmente (Montessori, 2016a). Un aspecto crucial dentro del aula Montessori son los materiales didácticos, que presentan tres características particulares: (1) permitir a los niños auto- 
educarse sin la necesidad de un adulto; (2) ser sistemáticos, continuos y específicos en un aspecto del desarrollo; y (3) poseer el control del error, permitiendo a los niños identificar los errores y corregirse a sí mismos. (Montessori, 2008; Montessori, 2016a).

La maestra Montessori tiene un rol pasivo en la educación de los niños y debe estar capacitada para observar adecuadamente el comportamiento de cada uno de ellos, saber diferenciar las manifestaciones espontáneas constructivas de la conducta inadecuada (Montessori, 2016c). Debe preparar un ambiente atractivo para los niños, que los cautive a realizar sus actividades. Además, ella misma debe ser capaz de cautivar a los niños para el trabajo. Dentro de las características que debe poseer se encuentra ser digna, un símbolo de autoridad y capaz de dirigir el aula y cuidar de ella. Debe ser el apoyo para los niños y crear un entorno de confianza, pero evitar que el apego se dirija hacia ella, si no, los niños no podrán desarrollar la independencia. Los niños deben dirigir su apego hacia los materiales (Montessori, 2016a).

\subsubsection{Sentido de Convivencia en Montessori}

Un aspecto importante en la educación Montessori es el desarrollo de la moral, educando para el respeto hacia los demás. Esto se ejemplifica en la libertad que tienen los niños, donde sus manifestaciones espontáneas son respetadas por todos. El principio moral más básico para Montessori es el compromiso del individuo hacia el grupo, el cual es aceptado por los niños (Montessori, 2017b). Por este motivo, en Montessori los círculos tienen un sentido: la solidaridad. Además, estos son importantes para la comunidad, pues los niños deben dejar de realizar sus actividades para participar. Dicha urgencia se podría explicar desde el argumento de que, al salvaguardar el bienestar colectivo, también se está priorizando el bienestar propio, entendido como democracia (William \& Keith, 2000). Es por ello que Montessori señala la resolución de conflictos como "axiomas de la paz", que busca enseñar a hablar honestamente, sin insultar ni culpar y escuchar sin interrumpir (Duckworth, 2006).

\subsubsection{Visión de los Padres}

Dado el rol de la educación y la perspectiva sobre los niños en la educación Montessori, es necesario que los padres mantengan esta coherencia en sus casas. Se les solicita que se mantengan al margen de los niños y aprendan a observarlos en su actividad, sin interferir ni ayudar cuando no se ha solicitado. Es importante que respeten los tiempos de sus hijos, para que ellos aprendan a respetar a los demás. Los adultos deben ofrecer otra actividad para el niño cuando se deba corregir una conducta (Montessori, 2017a). Esto no les quita la autoridad a los padres, sino que se enfoca desde otro punto de vista. Los niños son quienes construyen su aprendizaje y los padres deben ayudar y colaborar en su obra. La autoridad y dignidad de los padres se mide en relación a cómo ellos favorecen el desarrollo de sus hijos (Montessori, 2015).

La presente investigación tiene como objetivo comprender cómo se construye la convivencia escolar en un ambiente de educación Montessori. Para responder a esta pregunta se desea conocer cómo se educa para la resolución de conflictos y qué aspectos los niños desarrollan que favorezcan una adecuada convivencia escolar en la comunidad educativa. Además de ello, se busca caracterizar las relaciones de convivencia entre los niños y los diferentes miembros de la comunidad, a través de sus percepciones de dicho proceso. 


\section{MATERIALES Y MÉTODOS}

Esta investigación se sitúa desde el paradigma cualitativo-constructivista, ontológicamente relativista, es decir, la realidad existe en forma de construcciones múltiples, fundamentadas social y empíricamente, locales y específicas, que dependen en su forma y contenido de las fuentes de información. Desde el punto de vista de los constructivistas, Krause (1995) plantea que las realidades múltiples son construidas a través del lenguaje, coincidiendo así con las "comunidades discursivas". Desde el enfoque metodológico se diseñaron etapas, con predominancia cualitativa, para facilitar la ejecución de la investigación.

\subsection{PARTICIPANTES}

Como criterio emergente de selección sobre la escuela a estudiar, el equipo de investigación revisó los antecedentes de las Escuelas Montessori de la comuna de Valparaíso, Chile. Se buscó seleccionar aquella con mayor trayectoria y reconocimiento, tanto por el Consejo Nacional de Educación de Chile como por la Asociación Internacional Montessori. El establecimiento seleccionado es un colegio particular con niños de 3 a 6 años para educación pre-básica y de 6 a 12 años para educación básica, separados en dos grupos denominados "Casa de Niños" y "Taller", respectivamente. Este último se subdivide, en relación al desarrollo de los estudiantes, en "Taller Menor" y "Taller Mayor". Para esta investigación, los estudiantes de Taller fueron invitados a participar de forma voluntaria, autorizados por sus padres mediante consentimiento informado, logrando 92\% de participación de los estudiantes. También se invitó al Equipo Pedagógico permanente, el cual se compone por una directora académica, maestros Montessori y asistentes, quienes participaron de forma voluntaria, expresado a través de un consentimiento informado, obteniendo un $86 \%$ de participantes del Equipo Pedagógico.

\subsection{DISEÑO}

Esta investigación cualitativa se dividió en dos etapas. La primera consistió en una entrevista de grupos focales, donde participaron los estudiantes de Taller Mayor y Taller Menor, con el objetivo de generar un espacio para que surjan actitudes, sentimientos, creencias, experiencias y reacciones en los participantes, para obtener una multiplicidad de miradas y procesos emocionales dentro del contexto del grupo (Escobar \& Bonilla-Jiménez, 2006). En la segunda etapa, se realizó un encuentro dialógico con el equipo pedagógico permanente, que tuvo por objetivo compartir los hallazgos emanados de los grupos focales de los estudiantes, de este modo se logró materializar la co-construcción del conocimiento.

\subsection{RECOLECCIÓN DE DATOS}

En esta instancia, el equipo de investigación diseñó una tabla de especificaciones técnicas, considerando los principales criterios emanados de investigaciones que permiten comprender, de manera flexible y emergente, los elementos de convivencia que construyen las comunidades Montessori, las cuales se rigen por los principios que estableció María Montessori. Se realizaron dos entrevistas de grupos focales, lideradas por un moderador y acompañado de un dibujante de interacciones (Noaks \& Wincup, 2004, citado en Escobar \& Bonilla-Jiménez, 2006). Con el fin de realizar un trabajo riguroso, se entrenó en la 
técnica de grupos focales al moderador y al dibujante, quienes son miembros del equipo de investigación, a través de un pilotaje con estudiantes universitarios. El fin del entrenamiento es cumplir con los criterios de rigurosidad del moderador, donde se exige lograr mantener la discusión en un solo tema y ritmo, involucrar a los individuos en la conversación y establecer un ambiente de confianza que elimine las barreras de la comunicación (Beck et al., 2004, citado en Escobar \& Bonilla-Jiménez, 2006). La entrevista de grupos focales fue grabada y transcrita literalmente. Para resguardar la identidad de los sujetos, durante la transcripción los nombres mencionados fueron sustituidos por nombres ficticios. Posteriormente, se realizó el encuentro dialógico con el equipo pedagógico de la escuela, donde se les expuso los hallazgos preliminares de los datos obtenidos de los grupos focales. En dicha instancia, se les realizaron preguntas acerca de las temáticas expuestas, con el fin de provocar un diálogo entre los investigadores y los profesionales de la escuela. El diálogo fue transcrito en la misma instancia para su posterior análisis. Al igual que durante la transcripción de los grupos focales, todo nombre mencionado fue reemplazado por uno ficticio.

\subsection{ANÁLISIS DE DATOS}

A partir de los audios transcritos, se consideró como unidad de análisis a todas las acciones verbales inteligibles captadas de los niños entrevistados. Estas unidades fueron identificadas, clasificadas, sintetizadas y agrupadas, para posteriormente levantar categorías analíticas, a través de una codificación inductiva. En el proceso de sistematización y reducción de datos se utilizó el software Nvivo® ${ }^{\circledR}$ 12, en el cual se elaboró un árbol de codificación que permitió establecer las dimensiones y categorías presentes en los grupos focales y encuentro dialógico. La transformación de datos procura cumplir el criterio de pertenencia de cada categoría utilizada en el árbol de codificación, siendo coherente con el paradigma constructivista de esta investigación.

\subsection{OBTENCIÓN Y VERIFICACIÓN DE RESULTADOS Y CONCLUSIONES}

Desde el enfoque interpretativo se utilizó la triangulación de investigadores, dado que los análisis del fenómeno de Convivencia Escolar fueron llevados a cabo por diferentes integrantes del equipo de investigación. Para obtener diversidad de visiones de los hallazgos, los investigadores provienen de distintas disciplinas. De esta manera, se reducen los sesgos que se pueden generar al utilizar un único investigador en la recolección y el análisis de datos, además esto agrega consistencia a los hallazgos (Okuda \& Gómez-Restrepo, 2005).

\section{RESULTADOS}

En este apartado, se presentan los hallazgos que emergieron durante las dos etapas de investigación: entrevistas de grupos focales y encuentro dialógico.

\subsection{GRUPOS FOCALES}

Durante el proceso de categorización, se reconocieron diversos elementos que se agruparon en dos dimensiones: (1) Convivencia entre niños y familia, que reúne elementos de la 
interacción entre los niños y sus familias sobre sucesos vinculados a la escuela y su educación; y (2) Convivencia entre niños, referido a la interacción entre los niños y los hechos cotidianos en la escuela.

\subsubsection{Convivencia entre niños y familia}

Las interacciones agrupadas dentro de esta dimensión fueron categorizadas como: Comunicación con la familia. Se define como las manifestaciones de los niños a sus familias sobre sus intereses y su quehacer escolar. Esta interacción no siempre es de su agrado, tal como se evidencia en las siguientes declaraciones:

(...) me preguntan todos los días, pero a veces yo ya estoy 'chato'2 (...) y me empiezan a preguntar cosas, entonces yo ya no digo nada, o a veces le digo todo lo que pasa, pero normalmente le digo todo lo que pasó. (Niño de Taller Mayor).

(Niño da un ejemplo de conversación) hicimos tal cosa y aprendí esto que me gustó y esto no, lo pasé mal porque 'no sé’ tuve un problema, pero ya lo resolvimos. $Y$ usualmente a veces como que no me lo preguntan tanto, a veces, siempre en el día me lo preguntan una vez, pero después no lo hablamos, no nos metemos demasiado en ese tema. (Niño de Taller Mayor).

\subsubsection{Convivencia entre niños}

Esta dimensión agrupa las interacciones en las siguientes categorías: Cooperación entre Niños, Resolución de Conflictos y Control de Interacciones.

\subsubsection{Cooperación entre niños}

Se entiende como una colaboración brindada a otros, considerando el contexto, con el fin de mejorar una situación. La ayuda presenta diversos focos, dentro de los cuales destacan tres. El primer caso es la ayuda colaborativa centrada en el conocimiento, donde existe una contribución bidireccional, con el fin de socorrer a otro en una situación relacionada con el conocimiento, como se manifiesta a continuación:

Como que nos auto-ayudamos. De memoria completamente no sé, pero creo que algunas veces, algunas veces algo... creo que ayudé una vez al Felipe y él también me ayudó, no me acuerdo en qué era, pero parece que era algo de ¿matemáticas? Geometría y después él me ayudó en... ¿era ortocentro? En ortocentro y él me dijo como se hacía porque yo no entendí mucho la presentación y después lo entendí y... bien. (Niña de Taller Mayor).

Otro foco es la ayuda centrada en la idea hacia otro, donde existe una colaboración brindada a otro considerando lo mejor para él. Esto se observa en la siguiente declaración

Expresión de cansancio.

Alude a un evento sin importancia. 
de un niño acerca del concepto que tiene de su par: "que es bueno para el otro o... ayudar también es como... claro como guiar al otro a un camino, eso es". (Niño de Taller Menor).

Finalmente, el último foco es la ayuda centrada en la confianza, como se observa a continuación: "Yo creo que ayudar es darle la confianza a otra persona de que tú puedes colaborar en algo de que la otra persona necesite”. (Niño de Taller Mayor).

\subsubsection{Resolución de conflictos}

Corresponde a las interacciones donde se observan conflictos y su resolución. Se ejemplifica una situación de conflicto declarada a continuación: "El Juan me dice niña mocosa .. jA mí también! (otra niña comenta)... se te ven los mocos y esas cosas" (Niño de Taller Menor).

La resolución de conflicto comprende diversas estrategias para solucionar estas situaciones. La escuela entrega la máxima importancia a resolver un conflicto, razón por la que los estudiantes dejan de hacer sus actividades para acudir a ello. En estas situaciones, se utiliza recurrentemente una estrategia llamada Círculo de Emergencia.

Encuentro que son los círculos de emergencias, es que por ejemplo nosotros estamos así como... acabamos de terminar salón y estamos preparando el almuerzo, poniendo las mesas y de repente como la guía dice como círculo de emergencia, así, pero no lo dice como gritando (...) todos se reúnen en el salón central y ahí...pero es como emergencia (expresando importancia), o sea uno no se puede quedar ahí como ya voy en 5 minutos iré. (Niño de Taller Mayor)

En las interacciones de los niños se registran tres formas de resolución de conflicto: arbitraje, mediación y diálogo, como se puede apreciar a continuación:

Arbitraje.

$Y$ los escuchó que me estaban diciendo 'Heidi'4 y dijo... dijo que pararan de decirme 'Heidi' y pararon (Niña de Taller Menor).

Mediación.

(...) tenemos por ejemplo un problema con alguien entonces, y no podemos resolverlo por nuestra cuenta, se lo explicamos, vamos y le explicamos el problema al guía y así ver... y ahí, a través de una conversación se ve cómo puede, se puede resolver el problema (Niño de Taller Mayor).

Diálogo.

Yo al menos lo resuelvo tratando de hablar con esa persona y si es que... a mí me pasa como que cuando tengo un conflicto con alguien y yo quiero hablar con esa persona, me cuesta mucho hablar con esa persona (...) como que todo se resuelva a partir de las palabras, entonces como primero así perdonar y luego seguir hablando para que se acabe el tema, porque puede haber sido un mal entendido (Niño de Taller Mayor).

Sobrenombre con intención de provocar. 


\subsubsection{Control de interacciones}

Esta categoría alude a la forma espontánea que tienen algunos de los niños de guiar las intervenciones de sus compañeros, lo que varía según el contexto. Esto se evidencia en las siguientes declaraciones:

No te han dado la palabra Gustavo (exclama el niño) (...) ¿Por qué otras personas hablan si no le han dado la palabra? (Niño de Taller Menor).

¡El cojín lo trajimos para que se sentaran, no para que lo pusieran en los brazos! (Niño de Taller Menor).

\subsection{ENCUENTRO DIALÓGICO}

Las declaraciones del personal pedagógico fueron categorizadas en cuatro dimensiones: (1) Aspectos del desarrollo de los niños; (2) Características del maestro Montessori; (3) Aspectos vinculados a la familia; y (4) Resolución de conflictos.

\subsubsection{Aspectos del desarrollo de los niños}

Esta dimensión involucra las declaraciones con respecto al conjunto de habilidades, actitudes y conocimientos que los niños desarrollan durante su trayectoria en la escuela. Este desarrollo se caracteriza por ser continuo y permanente. Se mencionan tres aspectos importantes sobre este desarrollo, las cuales son autonomía, adaptabilidad y autoestima.

\subsubsection{Autonomía}

Comprende la capacidad que tiene el niño de establecer sus propias concepciones y orientarse por ellas a la hora de tomar decisiones. Esto se puede observar en la declaración del equipo pedagógico:

No deberíamos hacer nada que el niño no pueda hacer. (Equipo Pedagógico).

El desafío está en poder acompañar al niño en su desarrollo de autonomía. (Equipo Pedagógico).

\subsubsection{Adaptabilidad}

Se refiere a la capacidad de los niños de cambiar aspectos propios frente a una situación que, por lo general, suele ser adversa y es convertida en una situación neutral o favorable para ellos. Esto puede observarse en la declaración del equipo pedagógico sobre los niños de la escuela:

Los niños deben ser cambiantes, para enfrentarse a los cambios (Equipo Pedagógico).

El caso de Carolina (...) donde ella sola buscó el manual de convivencia, lo leyó y tuvo la capacidad de adaptarse a las normas del nuevo Liceo (refiere a una egresada de la 
Escuela Montessori que se inserta a la secundaria en un sistema de educación tradicional) (Equipo Pedagógico).

\subsubsection{Autoestima}

Esta categoría comprende las declaraciones sobre la valoración, con un enfoque positivo, que tiene el niño sobre aspectos propios, tal como se ejemplifica en la siguiente declaración: "Los niños deben poseer una adaptabilidad, y autoestima consistente" (Equipo Pedagógico).

\subsubsection{Características del maestro Montessori}

Esta dimensión registra las características que debe tener el maestro Montessori y el rol que debe cumplir. Se obtuvieron dos categorías: Enfoque reflexivo y Foco centrado en el niño.

\subsubsection{Enfoque Reflexivo}

Es una característica propia y permanente del personal pedagógico, la cual surge por situaciones de cuestionamiento sobre su quehacer docente dentro del Método Montessori y las decisiones que se deben tomar frente a las interacciones entre los niños. Esto se demuestra en la siguiente declaración:

A veces es un camino doloroso porque no siempre sabemos acompañar al niño, estamos en reflexión permanente, es muy trabajoso. El guía requiere perdonarse todos los días. Está todo el cuestionamiento de quien no tiene recetas (refiriéndose a un método de enseñanza-aprendizaje) Es un cuestionamiento que todos vivimos, una situación autorreflexiva permanente. (Equipo Pedagógico).

\subsubsection{Foco centrado en el niño}

Corresponde a un cambio de disposición en el rol de la escuela, opuesto a la educación tradicional. El niño es el protagonista de su propio aprendizaje y eje central del quehacer del equipo pedagógico, tal como se observa a continuación:

Los niños tienen un plan íntimo, lo principal es entender que no nos pertenece (Equipo Pedagógico).

(...) Construcción en la que los niños se sientan protagonistas (Equipo Pedagógico).

Ofrecer al niño lo que necesite independiente del contexto, nuestra labor no se puede ver comprometida por el contexto, ofrecemos lo mismo a todos los niños (Equipo Pedagógico).

\subsubsection{Aspectos vinculados a la familia}

Esta dimensión reúne las declaraciones sobre la interacción de la escuela y su personal con las familias, las cuales se dividen en: Interés de la familia y Confianza con el rol del maestro. 


\subsubsection{Interés de la familia}

Se refiere a la importancia que los padres entregan con respecto al Método Montessori y a la escuela como tal. Se declara un interés pasivo por parte de los padres, tal como se puede ver a continuación.

Si, si hay un interés declarado por las familias, pero que no se ve en la realidad. Dos veces al año se comparten materiales a los padres. Los guías les presentan el material como si fueran niño (haciendo referencia a unas Jornadas de Padres) (Equipo Pedagógico).

Los padres se encuentran en un proceso de limpieza de una tradición, de entender qué es una Escuela Montessori, qué implica acompañar en un sistema distinto. Se necesita un apoyo mayor, porque son requerimientos distintos (Equipo Pedagógico).

\subsubsection{Confianza en el rol del maestro}

Corresponde a las declaraciones sobre la seguridad que da la familia al personal pedagógico en su rol de maestro. Para apoyar esta confianza, el personal pedagógico utiliza estrategias de recopilación de información que los padres pueden consultar.

A los padres les tranquiliza saber que los guías llevan un registro y que pueden consultarlo. Hay una dualidad, quieren saber y es muy distinto a lo que ellos conocen. Hacen voto de confianza. (Equipo Pedagógico).

\subsubsection{Resolución de conflicto}

Esta dimensión corresponde a las estrategias que utiliza la escuela para resolver conflictos. Se realizaron dos categorías a partir de los hallazgos encontrados: Resolución centrada en el otro y Modalidades de círculo de emergencia.

\subsubsection{Resolución centrada en el otro}

Alude a la búsqueda de un acuerdo entre las partes en conflicto, enfocado en las sensaciones y necesidades, además de la colaboración con los otros. Esto se ejemplifica en la siguiente declaración.

(Comentando una situación ocurrida anteriormente en la Escuela) Mauro botó jugo y Matías se burló de él. Antecedentes de Matías: no es primera vez que lo hace, entonces le pregunto ‘¿Qué pasó? y ¿Qué hiciste? ¿Cómo crees que puedas solucionarlo? A mí se me va a ocurrir una solución, tienes que prepararle la colación a Mauro'. La energía que ocupaste en burlarte, la empleas en proveer. Mauro se sintió querido y protegido por Matías. (Equipo Pedagógico). 


\subsubsection{Modalidades de círculo de emergencia}

Se trata sobre las formas de ejecutar la estrategia de resolución de conflicto llamada Círculo de Emergencia, adoptado por la escuela, donde se mezclan los círculos de paz con otras estrategias de resolución de conflictos ya mencionadas. Esta se puede realizar en pareja o de forma grupal y en diferentes grados de complejidad. A continuación, se ejemplifica el uso de esta estrategia.

Lo que convoca círculo de emergencia es una situación que daña al grupo. Tiene mayor efecto si tú dices que el salón funciona (...) hacemos esto, esto y esto. De este modo ya todos saben quién 'metió las patas'. Cuando está más claro se habla de forma individual, uno a uno, por lo general, eso es con los niños más pequeños (Equipo Pedagógico).

Se les da la opción a los niños de expresar cómo se sintió en esa situación, en un conflicto de a dos se resuelve pidiendo disculpas, si no se llega a acuerdo entre los niños el guía da una propuesta y la aceptan (Equipo Pedagógico).

\section{DISCUSIÓN Y CONCLUSIÓN}

Analizando los datos recogidos en ambos grupos se observa correspondencia y coherencia. En cuanto a la autonomía, esta es mencionada como uno de los aspectos que los niños deben desarrollar, la que es definida por el equipo pedagógico por la escuela investigada como la capacidad para establecer concepciones propias y poder tomar decisiones acordes a estas. Esta definición de autonomía se relaciona con la visión de libertad de Montessori, que se concibe como la capacidad de identificar lo que se necesita y realizar las acciones para conseguirlo (Montessori, 2015). Coherente a ello, algunos autores concuerdan definir la autonomía como la capacidad de realizar juicios propios sobre cómo actuar y guiar una auténtica motivación intrínseca (Muñiz, 2009). Frankfurt (1971) define la libertad bajo dos conceptos: Libertad de acción y Libertad de la voluntad. Esta última está relacionada con la autonomía, dado que el individuo apoya sus deseos bajo una autoevaluación reflexiva, priorizando sus necesidades. En esta investigación, la autonomía es observada cuando los niños hablan sobre la ayuda a otros, donde la perciben como un acto de confianza, ofreciendo sugerencias, pero permitiendo que la otra persona pueda decidir aceptarlas o desecharlas. También, se aprecia que el control de la interacción durante el grupo focal evidencia que los niños son capaces de recurrir a su propio criterio para controlar la situación, sin esperar la participación de adultos.

Un aspecto señalado por los niños es la cooperación entre ellos, en donde lo entienden como una ayuda que se brindan mutuamente para mejorar una situación. Este hallazgo es coherente por lo postulado con respecto con el desarrollo de la moral de la educación Montessori, que se enfoca en el respeto por los demás (Montessori, 2017b). La ayuda señalada por los niños, centrado en el otro y en la confianza, está relacionada con el compromiso del individuo hacia el grupo esperado en esta filosofía educativa.

Chilenismo referido a cometer un error. 
La adaptabilidad de los niños frente a situaciones adversas es otro concepto que emerge por parte del equipo pedagógico de la escuela investigada. Un desarrollo adecuado de la capacidad de adaptabilidad de los niños está directamente relacionado con mayores habilidades sociales (Brock \& Curby, 2016). En el encuentro dialógico, los maestros Montessori declaran buscar que los niños desarrollen la adaptabilidad mediante la convivencia en la comunidad, donde a partir de situaciones adversas puedan llegar a una solución favorable, como puede observarse en las situaciones de conflicto y las estrategias utilizadas, tales como el arbitraje, mediación y el diálogo, considerando que son los niños los principales participantes de estas estrategias en el aula Montessori. Esto se refleja en el uso del círculo de emergencia, referido por la entrevista de grupo focales con los niños, como instancia social para tratar y resolver conflictos en la comunidad estudiada. El uso de estas estrategias restaurativas permite desarrollar las habilidades sociales y valorar las instancias como un proceso de aprendizaje (Morrison \& Vaandering, 2012). La autoestima es otro concepto aludido por el equipo pedagógico de la escuela Montessori. En los grupos focales se puede inferir que, durante el control de las interacciones, los niños muestran una postura reguladora durante la interacción y la expresan sin temor a recriminaciones en su entorno social.

En relación al enfoque reflexivo, se observa una coherencia entre lo planteado por el equipo pedagógico y las acciones mencionadas por los niños en torno a la convivencia escolar, especialmente con respecto al conflicto. Los indicadores de esta correspondencia se observan en la toma de decisiones y las estrategias a aplicar en el momento en que se produce un conflicto que los niños no pueden resolver por sí mismos. La toma de decisiones se relaciona con las características del maestro en el Método Montessori, ser alguien de respeto y un símbolo de autoridad, lo que le permite dirigir y cuidar el aula (Montessori, 2016a). El foco en la educación Montessori son los niños, considerados los constructores del hombre y, por ende, de su conocimiento (Montessori, 2015). Esto se refleja en las declaraciones del equipo pedagógico de la escuela, donde mencionan que el niño es el protagonista de la construcción de su conocimiento. Durante los grupos focales, los niños mencionan a sus maestros en momentos anecdóticos, relacionados a situaciones de conflicto; durante la presentación y apoyo en ciertos temas o con materiales, pero no como agentes que dirigen y deciden su aprendizaje. Esto es resultado del rol pasivo del maestro y su misión como observador de su comportamiento y desarrollo, siendo capaz de guiar a los niños a la concentración en la actividad, donde son ellos mismos los constructores de su aprendizaje (Montessori, 2016a; Montessori, 2016c).

En relación al conflicto, los estudiantes optan por utilizar la estrategia del diálogo para resolverlas, donde sólo participan las partes en conflicto y son ellos mismos quienes buscan llegar a un acuerdo, tal como lo mencionan Morrison y Vaandering (2012). En casos donde esto no es posible, los niños deciden llegar a otras instancias donde un tercero, como los maestros, puede apoyar la resolución. Dentro de las declaraciones de los niños, se menciona que la estrategia utilizada es el círculo de emergencia, cuyo modelo es explicado en la Figura 1. Esta estrategia demuestra la toma de medidas restaurativas para la resolución de los conflictos, las cuales tienen por objetivo reparar el daño y crear oportunidades, además de fomentar la democracia y enriquecer los lazos sociales (Morrison \& Vaandering, 2012). Esto es coherente con la importancia del compromiso del individuo hacia el grupo que declara Montessori en sus postulados (Montessori, 2017b). Bajo esta visión, los conflictos deben solucionarse a través de la conversación honesta y transparente, decir lo que se piensa y escuchar a los demás, tomando estos elementos como axiomas de la paz (Duckworth, 2006). 
Figura 1. Modelo de Resolución de Conflicto "Círculo de Emergencia". Demuestra las decisiones del equipo pedagógico sobre la estrategia de resolución de conflictos que se implementará durante un círculo conformado espacialmente por los integrantes de la comunidad. A modo de escalera ascendente, se muestra el nivel de independencia que tienen los niños para tomar y acordar las decisiones para llegar a una solución. Con diferencia de tonalidad se muestra el nivel de desarrollo de los niños en torno a qué estrategia utilizan ellos.

\section{Modelo de Resolución de Conflictos}

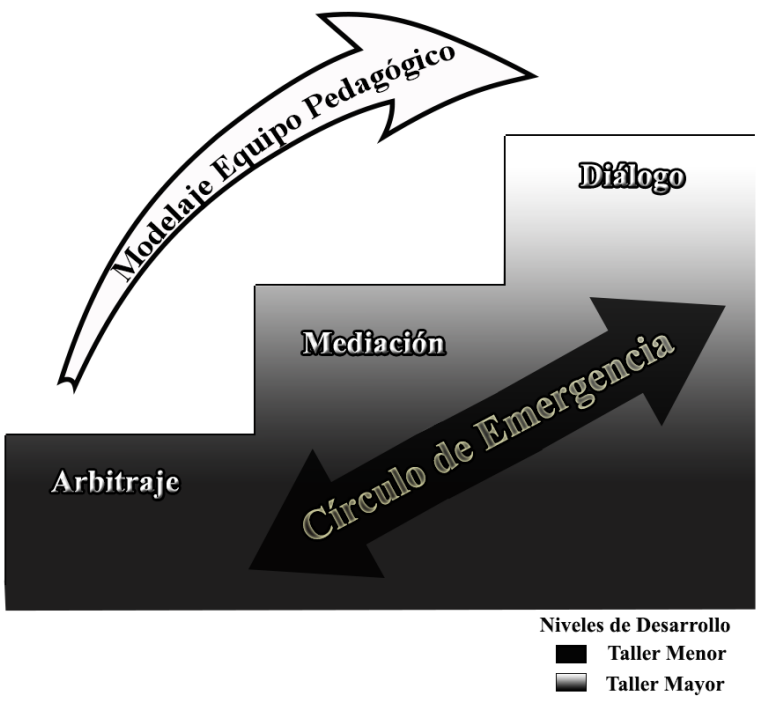

(Fuente: elaboración propia).

En relación a la familia, se menciona la confianza que tienen los padres acerca del equipo pedagógico. Para fomentar esta relación, se otorga el acceso a los padres al registro del desarrollo de los niños. Esto se contrapone con lo declarado por los niños, quienes mencionan ser interrogados frecuentemente por sus familias, sobre sus actividades, lo que no les agrada. Además, este último hecho se contradice con la actitud que la educación Montessori espera de los padres, donde deben respetar a los hijos, darles tiempo y ser un participante pasivo de la autoconstrucción de ellos mismos (Montessori, 2017a). Según la evidencia entregada por el equipo pedagógico, esto puede producirse por un desconocimiento o confusión de los padres con respecto a la educación Montessori y experiencias en sistemas de enseñanza de transmisión-recepción. A raíz de esto, se propone un modelo sobre cómo la escuela Montessori investigada genera elementos para desarrollar la confianza de los padres en el enfoque Montessori (Figura 2). 
Figura 2. Se muestra el modelo aplicado en la escuela para desarrollar la confianza de la familia en el enfoque Montessori. En el camino en negro, se observan las acciones efectuadas por la escuela de forma progresiva para construir la confianza, desde la Relevancia de la Observación como un primer paso, hasta el Rol Centrado en el Acompañamiento del Niño, como último proceso. Además, en los carteles grises a un costado del camino, se observa el proceso emergente de los padres, que desarrollan a través de estas etapas, de forma progresiva para llegar a la construcción de la confianza.

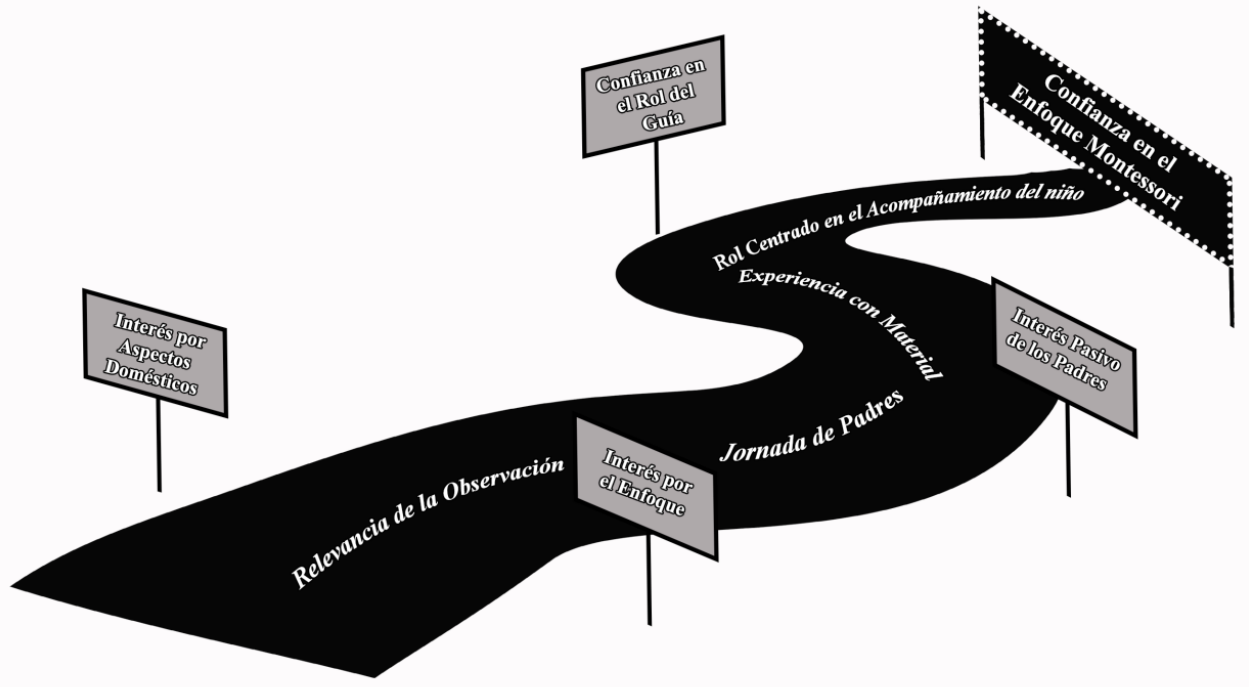

(Fuente: elaboración propia).

En conclusión, el círculo de emergencia como estrategia pedagógica para la resolución de conflictos es coherente con el desarrollo integral, centrado en las sensaciones y necesidades de los niños. Se han recogido aspectos empíricos que pueden ser replicados en establecimientos del sistema tradicional de educación. Entonces, ¿cómo poder mejorar la convivencia escolar en ambientes tradicionales?, ¿podrían estos hallazgos guiar la construcción de un clima escolar nutritivo? Esta investigación aporta evidencia que apoya que niños de edades tempranas pueden desarrollar competencias que permiten la resolución de conflictos. Además, el desarrollo de estas habilidades sociales permite formar seres íntegros, enfocados en la sana convivencia. El desafío del siglo XXI se centra en mejorar la convivencia en las escuelas del mundo, mediante la educación para la paz, implementando estrategias restaurativas concretas como lo hace la escuela Montessori estudiada mediante el círculo de emergencia y el desarrollo integral del individuo. 
Estudios Pedagógicos XLVII N 2: 299-317, 2021

¿CÓMO SE CONSTRUYE LA CONVIVENCIA ESCOLAR EN LA EDUCACIÓN MONTESSORI? ESTUDIO DE CASO DE

UNA ESCUELA MONTESSORI DE VALPARAIISO, CHILE

\section{REFERENCIAS BIBLIOGRÁFICAS}

Acosta, A. (2004). Regulación de conflictos y sentimientos. En B. Molina \& F. Muñoz (Eds.) Manual de paz y conflictos (pp. 145-169). España: Universidad de Granada.

Agencia de la Calidad de la Educación (2013). Clima de convivencia escolar según estudiantes de II medio. Apuntes sobre la calidad de la Educación, Año 1: $\mathrm{N}^{\circ} 10$. Santiago: Autor.

Armour, M. (2013). Ed White Middle School Restorative Discipline Evaluation: Implementation and Impact, 2012/2013 Sixth Grade. http://sites.utexas.edu/irjrd/files/2016/01/Year-2-Final-EWReport.pdf

Aron, A., Milicic, N., Sánchez, M. \& Subercaseaux, J. (2017) Construyendo juntos: Claves para la convivencia escolar. Santiago, Agencia de Calidad de la Educación.

Badiei, F. (2010, enero). Online arbitration definition and its distinctive features. En M. Poblet, M. Abrahams \& J. Zeleznikow (Eds.). Proceedings of the 6th international workshop on online dispute resolution (ODR Workshop'10) (pp. 87-93), Liverpool, UK. Spain: Universitat Autònoma de Barcelona.

Brock, L. \& Curby, T. (2016). The role of children's adaptability in classrooms characterized by low or high teacher emotional support consistency. Social Psychology Review, 45(2), 209-225. https://doi.org/10.17105/SPR45-2.209-225

Carrasco, C., Ascorra, P., Lóprez, V. \& Álvarez, J. (2018). Tensiones normativas de los fiscalizadores de la Superintendencia de Educación en la(s) política(s) de convivencia escolar en Chile. Perfiles Educativos, 40(259), 126-143.

Duckworth, C. (2006). Teaching peace: a dialogue on the Montessori Method. Journal of Peace Education, 3(1), 39-53. https://doi.org/10.1080/17400200500532128

Escobar, J. \& Bonilla-Jiménez, F. (2006). Grupos focales: una guía conceptual y metodológica. Cuadernos hispanoamericanos de psicología, 9(1), 51-67.

Fabelo, T., Thompson, M. y Plotkin, M. (2011). Breaking schools' rules: A statewide study of how school discipline relates to students' success and juvenile justice involvement. New York: Council of State Governments Justice Center.

Frankfurt, H. (1971). Freedom of the will and the concept of a person. The Journal of Philosophy, 68(1), 5-50. https://doi.org/10.2307/2024717

Fronius, T., Persson, H., Guckenburg, S., Hurley, N. \& Petrosino, A. (2016) Restorative Justice in U.S. Schools: A Research Review. The WestEnd Justice \& Prevenion Research Center, San Francisco. http://jprc.wested.org/wp-content/uploads/2016/02/RJ_Literature-Review_20160217.pdf

Funes, S. (2000). Resolución de conflictos en la escuela: una herramienta para la cultura de paz y la convivencia. Contextos Educativos, (3), 91-106. https://doi.org/10.18172/con.466

Generalitat de Catalunya/Departament d'Ensenyament (2017). Projecte de convivència $i$ èxit educatiu: Continguts de l'aplicació informàtica per a l'elaboració del projecte de convivència. Catalonia: Autor.

Gellin, M. (2018). Mediation in Finnish Schools: From Conflicts to Restoration. En A. Nylund, K. Ervasti K. \& L. Adrian (Eds.). Nordic Mediation Research (pp. 247-266). Springer: Cham.

Gregory, A, Clawson, K., Davis, A. \& Gerewitz, J. (2016) The Promise of Restorative Practices to Transform Teacher-Student Relationships and Achieve Equity In School Discipline. Journal of Educational and Psychological Consultation, 26(4), 325-353. https://doi.org/10.1080/1047441 2.2014.929950

Krause, M. (1995). La investigación cualitativa: Un campo de posibilidades y desafíos. Revista Temas de Educación, 7(7), 19-40.

Montessori, M. (2008). Los Angeles lectures. En R. Buckenmeyer (Ed.) The California lectures of Maria Montessori (pp. 6-29). Amsterdam: Montessori-Pierson Publishing Company.

. (2013). Independence of the Child. En S. Feez (Ed.). The 1913 Rome lectures: First international training course (pp. 106-114). Amsterdam: Montessori-Pierson Publishing Company. 
(2015). La mente absorbente del niño. Amsterdam: Montessori-Pierson Publishing Company. . (2016a). The child, society and the world. Amsterdam: Montessori-Pierson Publishing Company.

. (2016b). The discovery of the child. Amsterdam: Montessori-Pierson Publishing Company. . (2016c). El método de la pedagogía científica: Aplicado a la educación de la infancia (6th ed.). Madrid: Biblioteca Nueva. .2017a). María Montessori le habla a los padres. Amsterdam: Montessori-Pierson Publishing Company.

(2017b) De la infancia a la adolescencia. Amsterdam: Montessori-Pierson Publishing Company.

Morrison, B. \& Vaandering, D. (2012). Restorative justice: pedagogy, praxis and discipline. Journal of School Violence, 11, 138-155. https://doi.org/10.1080/15388220.2011.653322

Muñiz, M. (2009). Autonomy as a foundation for human development: A conceptual model to study individual autonomy. MGSoG Working Paper, 2009-WP001, Maastricht. http://collections.unu. edu/eserv/UNU:929/wp2009- 011.pdf

Muñoz, F. (2004). Qué son los conflictos. En B. Molina \& F. Muñoz (Eds.) Manual de paz y conflictos (pp. 145-169). España: Universidad de Granada.

Muñoz, M. T., Lucero, B., Cornejo, C., Muñoz, P. \& Araya, N. (2014) Convivencia y clima escolar en una comunidad educativa inclusiva de la provincia de Talca, Chile. Revista electrónica de Investigación Educativa, 16(2), 16-32.

Okuda, M. \& Gómez-Restrepo, C. (2005). Métodos en investigación cualitativa: triangulación. Revista Colombiana de Psiquiatría, 34(1), 118-124.

Williams, N. \& Keith, R. (2000). Democracy and Montessori education. Peace Review, 12(2), 217 222. https://doi.org/10.1080/10402650050057861. 
